\title{
Low risk of serological cross-reactivity between dengue and COVID-19
}

\author{
Michele Spinicci ${ }^{1,2} /{ }^{+}$, Alessandro Bartoloni ${ }^{1,2}$, Antonia Mantella', \\ Lorenzo Zammarchi ${ }^{1,2}$, Gian Maria Rossolini ${ }^{1,3}$, Alberto Antonelli ${ }^{1,3}$ \\ ${ }^{1}$ University of Florence, Department of Experimental and Clinical Medicine, Florence, Italy \\ ${ }^{2}$ Careggi University Hospital, Infectious and Tropical Diseases Unit, Florence, Italy \\ ${ }^{3}$ Careggi University Hospital, Microbiology and Virology Unit, Florence, Italy
}

In the near future, the overlap of Coronavirus disease 2019 (COVID-19) and dengue epidemics is a concrete threat in tropical regions. Co-epidemics of COVID-19 and dengue could be an overwhelming challenge for health systems in low- and middleincome countries. In this work, we investigated potential serological cross-reactions between COVID-19 and dengue patients. Among 32 COVID-19 positive sera, no positive Dengue virus (DENV) IgG/IgM results were observed. On the other hand, one false-positive result was observed among 44 DENV-positive sera tested for COVID-19 antibodies with each of the two rapid tests used. Further data on accuracy of COVID-19 diagnostic test are urgently warranted.

Key words: COVID-19 - SARS-CoV-2 - dengue - antibodies

Since December 2019, Coronavirus disease 2019 (COVID-19), caused by severe acute respiratory syndrome coronavirus 2 (SARS-CoV-2), emerged in the international scene as a major public health concern. COVID-19 pandemic is having a disrupting impact on health systems throughout Asia, Europe and America.$^{(1)}$ At the same time, a large outbreak of dengue is ongoing in Latin American countries, with several deaths being recorded.(2)

In the near future, the overlap of COVID-19 and dengue epidemics is a concrete threat in tropical regions. . $^{(3,4)}$ COVID-19 and dengue share several features of clinical and laboratory presentation, and differential diagnosis should rely on specific diagnostic tests. ${ }^{(5)}$ Recently, two cases of false-positive results by rapid diagnostic test (RDT) for dengue in patients with SARS-CoV-2 infection were reported from Singapore. ${ }^{(6)}$ If serological cross-reactivity between patients with COVID-19 and dengue will be confirmed, it may result in a high number of misdiagnoses, with dangerous consequences both from the patients and from public health point of view..$^{(7)}$

In order to further explore this possibility, we investigated potential serological cross-reactions between COVID-19 and dengue patients, by performing commercial assays for detection of anti-Dengue virus (DENV) and anti-SARS-CoV-2 antibodies on sera from well-characterised COVID-19- and dengue-positive patients, respectively.

A total of 32 anonymised sera, obtained from symptomatic patients with COVID-19 diagnosed by positive anti-SARS-CoV-2 IgM/IgG (32/32, tested by 2019-

doi: 10.1590/0074-02760200225

Financial support: This article has been supported by funds of Ministry of Education, University and Research (Italy) Excellence Departments 2018-2022 (Project for the Department of Experimental and Clinical Medicine).

+ Corresponding author: michele.spinicci@unifi.it

(1) https://orcid.org/0000-0002-5377-7118

Received 10 May 2020

Accepted 04 August 2020
NCOV IgG/IgM Rapid Test Cassette, ScreenItalia, Perugia, Italy) and/or RT-PCR for SARS-CoV-2 on nasopharyngeal swab (26/32), were tested by Dengue Enzyme Linked Immune Assay (ELISA) DENV IgG-IgM (VIRCELL, Granada, Spain). Sera were collected and tested in Italy, at the Careggi University Hospital of Florence, where dengue is not endemic, and previous exposure to DENV was unlikely.

Moreover, 44 anonymised DENV-positive sera, from cases of acute dengue (27 of whom confirmed by NS1 antigen positivity, plus 17 probable diagnosis with only IgM and $\mathrm{IgG}$ positivity), were tested by using COVID-19 IgG/ IgM Rapid Test Cassette (Orient Gene, Zhejiang, China) and 2019-NCOV IgG/IgM Rapid Test Cassette (ScreenItalia, Perugia, Italy). All DENV-positive sera were collected from travellers, before SARS-CoV-2 emergence.

Among 32 COVID-19 positive sera, no positive DENV IgG/IgM results were observed. On the other hand, one false-positive result was observed among 44 DENV-positive sera tested for COVID-19 antibodies with each method (in one case for $\operatorname{IgG}$ and $\operatorname{IgM}$, and in another for IgG only), in two different samples.

Serological test for dengue are known to be affected by cross-reactivity issues in areas were multiple Flavivirus, such as Zika, Yellow fever or Japanese encephalitis virus are circulating. The risk of false-positive results is reduced when dengue $\operatorname{IgM} / \operatorname{IgG}$ testing is paired with NS1 antigen capture..$^{(8)}$

According with our results, the concern about falsepositive dengue serology in COVID-19 patients could be downsized, at least when an ELISA is used. On the other hand, COVID-19 false-positive results are possible in patients with DENV infection, although at a low rate and variably using different RDTs for COVID-19. It should be also considered that these COVID-19 falsepositive results may reflect different aspecific crossreactivity, not necessarily related to DENV-specific antibodies ${ }^{(9)}$ Of course, this is a preliminary study on a limited number of samples, which need to be validated on a wider population. 
In low- and middle-income countries, where arboviruses and other common tropical diseases are highly endemic, SARS-CoV-2 spread will represent an additional challenge for clinicians. ${ }^{(10,1)}$ In particular, co-epidemics of COVID-19 and dengue could be an overwhelming situation for health systems. ${ }^{(12)}$ Further studies are warranted to better elucidate the accuracy of commercially available RDTs for SARS-CoV-2 antibodies detection and their role within the strategy for COVID-19 diagnosis and control.

\section{AUTHORS' CONTRIBUTION}

$\mathrm{MS}, \mathrm{AB}, \mathrm{LZ}$ and GMR contributed to the study design; AA and AM performed laboratory tests. All authors contributed to data analysis, interpretation and critically reviewed the paper.

\section{REFERENCES}

1. WHO - World Health Organization. Coronavirus disease (COVID-2019) situation reports. 2020. Available from: https://www. who.int/emergencies/diseases/novel-coronavirus-2019/situationreports/ Last access: 23rd June 2020.

2. PAHO/WHO - Pan American Health Organization/World Health Organization. Epidemiological update: arbovirus. Washington, DC: PAHO/WHO; 2020

3. Navarro JC, Arrivillaga-Henríquez J, Salazar-Loor J, RodriguezMorales AJ. COVID-19 and dengue, co-epidemics in Ecuador and other countries in Latin America: pushing strained health care systems over the edge. Travel Med Infect Dis. 2020; 101656. [published online ahead of print].
4. Lorenz C, Azevedo TS, Chiaravalloti-Neto F. COVID-19 and dengue fever: a dangerous combination for the health system in Brazil. Travel Med Infect Dis. 2020; 35: 101659.

5. Lam LTM, Chua YX, Tan DHY. Roles and challenges of primary care physicians facing a dual outbreak of COVID-19 and dengue in Singapore. Fam Pract. 2020; cmaa047. [published online ahead of print].

6. Yan G, Lee CK, Lam LTM, Yan B, Chua YX, Lim AYN, et al. Covert COVID-19 and false-positive dengue serology in Singapore. Lancet Infect Dis. 2020; 20(5): 536.

7. Li R, Pei S, Chen B, Song Y, Zhang T, Yang W, et al. Substantial undocumented infection facilitates the rapid dissemination of novel coronavirus (SARS-CoV2). Science. 2020; 368(6490): 489-93.

8. Muller DA, Depelsenaire AC, Young PR. Clinical and laboratory diagnosis of dengue virus infection. J Infect Dis. 2017; 215(Suppl. 2): S89-S95.

9. Sethuraman N, Jeremiah SS, Ryo A. Interpreting diagnostic tests for SARS-CoV-2. JAMA. 2020; 10.1001/jama.2020.8259. [published online ahead of print].

10. Bokhari SMMA, Mahmood F, Bokhari SMSA. Case report: diagnosis of novel Coronavirus disease (COVID-19) versus tropical diseases in Pakistan. Am J Trop Med Hyg. 2020; 103(1): 77-8.

11. Joob B, Wiwanitkit V. COVID-19 can present with a rash and be mistaken for dengue. J Am Acad Dermatol. 2020; 82(5): e177.

12. Wu D, Lu J, Liu Q, Ma X, He W. To alert co-infection of SARS$\mathrm{COV}-2$ and dengue virus in developing countries in the dengueendemic area. Infect Control Hosp Epidemiol. 2020; 1. [published online ahead of print]. 